\title{
Clustering Organisms Using Metabolic Networks
}

\author{
Tomasz Arodź \\ Institute of Computer Science, AGH University of Science and Technology, \\ al. Mickiewicza 30, 30-059 Kraków, Poland \\ arodz@agh.edu.pl
}

\begin{abstract}
Topological properties of metabolic networks may reflect systematic differences between evolutionary distinct groups of organisms. Indeed, the mean shortest path length between metabolites is, on average, longer in eukaryotes than in bacteria. We show that not only the averages of groups differ, but the organisms can be successfully clustered, based on network properties, into categories corresponding to taxonomic groups. We use the fact that in metabolic networks of different organisms, correspondence between vertices is available. We compare our approach with several graph indices employed previously to analyse metabolic networks, and show that they fail at achieving level of clustering similar to ours. Finally, we show that the phylogenetic tree constructed using network-based approach agrees in most cases with gene-based phylogeny.
\end{abstract}

Keywords: Metabolic networks, Phylogenetic tree, Graph descriptors.

\section{Introduction}

Nature provides us with a stunning diversity of life forms. First taxonomic efforts aimed at classifying and bringing order to this heterogeneity relied on apparent phenotypic differences between organisms. With the advances in molecular biology, study of evolution of species found ground on the conservation and divergence of their corresponding gene or protein sequences. Phylogenetic analyses of evolutionary conserved genome units, such as $16 \mathrm{~S}$ rRNA, allowed for constructing trees that capture the evolutionary closeness of organisms, with more distant species occupying nodes in distant branches. Still, reliance in the construction of a phylogenetic tree on a specific sequence can be unstable, due to events such as horizontal gene transfer. As genome sequencing efforts progress, whole-genome phylogenetic approach emerged to circumvent this issue [1].

Analysing sequences of some or even all genes or proteins does not make explicit the system-level interactions between these entities. Recently, networkbased approach gained popularity in modelling and analysing the linked and entangled structure that turns these individual units into a living cell [2]. In particular, metabolic networks, linking metabolites through enzymes that catalyse reactions between them, were shown to belong to a class of complex networks with scale-free connectivity 3. Such networks are resilient to small random changes, which may accumulate through generations and give hints to the evolutionary distance between organisms.

M. Bubak et al. (Eds.): ICCS 2008, Part II, LNCS 5102, pp. 527 534, 2008.

(C) Springer-Verlag Berlin Heidelberg 2008 
The goal of the paper is to cluster organisms according to similarity of the topological properties of their metabolic networks. Using such networks to aid the construction of phylogenetic trees has been introduced recently. One approach for evaluating distance between organisms' networks relies on information other than topological. Measures of overlap between sets of metabolites [45] or enzymes 6 6 present in different organisms were used. Other methods use information on enzyme similarity instead or in addition to topological properties, which makes them more akin to classical phylogenetic algorithms that operate on genetic closeness. For example, enzyme functional class similarity was employed together with similarities of enzyme neighbourhoods [7]. Extent of sequence agreement of enzymes, and of those substrates that are proteins, was also used 8 .

Another group of methods relies only on topological properties of the graph, once the network structure of enzymatic connections linking metabolites has been inferred from the genome. Average shortest path lengths of metabolic networks was shown to be shorter for bacteria than for eukaryotes and archaea [9]. Average clustering coefficient and average betweenness centrality was proposed as means to discriminate archaea from bacteria, but it was validated using only 11 organisms [10]. In the same study, network diameter and average shortest paths were deemed not suitable for clustering. Phylogenetic trees were also constructed based on graph kernels [1]. Rank order of metabolites or enzymes was also used 4, revealing clustering of parasitic and non-parasitic bacteria, eukaryotes and archaea, but the networks involved spurious connections through abundant current metabolites, for example cofactors such as ATP.

We show here that analysing metabolic networks using only simple graph descriptors to infer similarity allows for obtaining meaningful phylogenetic results. We use the fact that network edges are spanned on a common set of vertices with known identities as metabolites. We calculate and compare vertex-specific descriptors, that is, topological properties for nodes common in networks of all organisms. Our approach does not involve quantitative measures of genetic or functional similarity of enzymes in the network. Nor does it use the differences in presence or absence of certain elements of the network, as we use descriptors for vertices that are present in all networks. Despite the simplicity of the topological descriptors used in calculating graph similarity, the resulting phylogenetic tree is in good accord with current phylogenetic knowledge.

\section{Methods}

Evaluation of similarity of two graphs is in most applications hindered by the lack of correspondence between elements of the network. Techniques to circumvent this involve dissimilarity measures based on the cost of edit operations required to transform one graph into another or rely on estimated vertex matching in graphs [12. Other methods compare graph indices, either global properties, such as graph diameter, or aggregated statistics of node- or edge-specific properties, such as average clustering coefficient [13. Also, more sophisticated functions of 
vertex-specific properties are in use, such as symmetric polynomials, which are invariant to the permutation of nodes [14].

In metabolic networks made publicly available by Ma and Zeng 9, which we analyse here, vertices represent well-defined chemical entities, such as glucose, while edges correspond to enzymatic reactions that transform the metabolites. Thus, the correspondence between vertices in the network is readily available. Permutation invariant measures or indices based on aggregate statistics are no longer necessary to obtain similarity between two graphs. Instead, we evaluate here an approach in which the values of vertex-specific topological properties are used directly to form a feature vector. Each feature corresponds then to a specific metabolite. Dissimilarities between metabolic networks are quantified by distances between corresponding feature vectors.

Metabolic networks were shown to be composed of a single giant connected component, and a set of isolated small components [15]. In further analysis, we have discarded these small components, and retained only the giant connected component of the network. One should note that in different organisms the whole networks, as well as the giant connected components, are of different size, and overlap only partially.

We have analysed two vertex-specific topological descriptors as basis for our feature vectors. A descriptor provides a single numerical value for each vertex in the network's giant connected component. One analysed property is the vertex degree, defined as the number of edges incident with the vertex. Alternatively, we analysed the betweenness centrality of a vertex $v$, defined as the fraction of the number of shortest paths between two vertices $s$ and $t$ passing through $v$ in the overall number of shortest paths between $s$ and $t$, averaged over all source-target pairs in the giant connected component.

Values of these descriptors are available only for nodes present in the network of a given organism, and also present in its giant connected component. To obtain feature vectors with no missing entries for all organisms, we identified vertices that are present in giant connected components of all the analysed metabolic networks. This resulted in a subset containing 100 nodes. We utilised the values of node degrees or node betweenness centrality only from these vertices. Thus, we arrive with 100-dimensional feature vectors that are well-defined across all the networks. Also, while the values are taken from only a subset of the connected component, they contain information from outside of the subset, as calculation of the vertex properties involves the whole giant connected component.

\section{Results and Discussion}

\subsection{Clustering Organisms Using Vertex-Specific Descriptors}

We have analysed metabolic networks of 44 organisms - 3 eukaryotes and 41 bacteria. The latter consist of 25 members of Proteobacteria, 12 Firmicutes and 4 Actinobacteria (see Fig. 4 for detailed information about the species analysed). For each organism, we obtained a feature vector of 100 values describing the vertex degrees of the nodes in its metabolic network. 


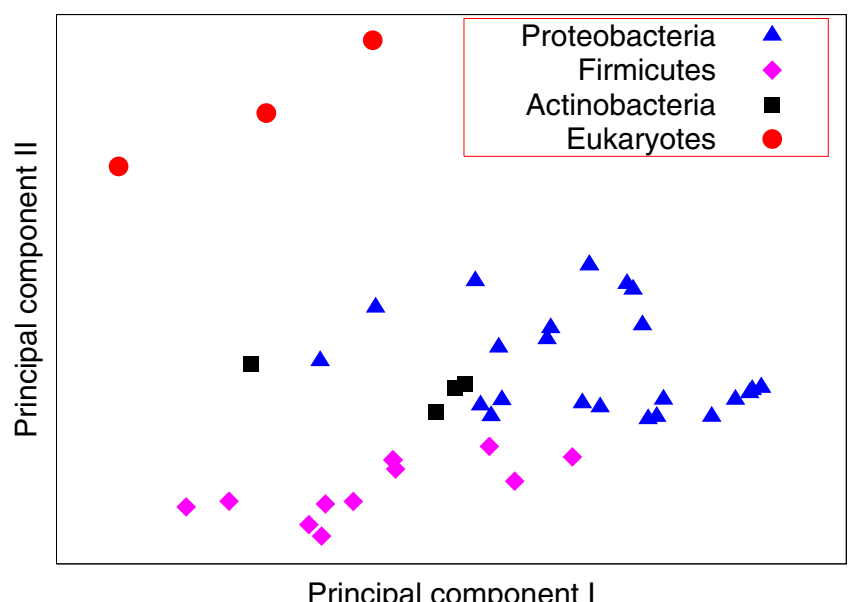

Fig. 1. First two principal components of the 100-dimensional feature space, formed by values of the vertex degrees of 100 vertices common to all giant connected components of the 44 analysed metabolic networks

In Fig. 1, we show the projection of vertex-specific descriptors into principal component space (PCA) [16, spanned by highest variance, mutually uncorrelated directions in the dataset. The figure indicates that metabolic networks of the three animals, as captured by the descriptors, are distinct from the networks of bacteria. Within the cluster of the latter, Proteobacteria form a region separated from Firmicutes. Actinobacteria are grouped between Frimicutes and Proteobacteria. The distances between the three animals are relatively large compared to distances between neighbouring bacterial organisms. This is consistent with the fact that the animals analysed, H. sapiens, D. melanogaster and C. elegans, represent different phyla, while within the phyla of Proteobacteria, Actinobacteria and Firmicutes several examples of each bacterial class are present.

\subsection{Comparison with Network Indices}

To compare the effectiveness of the vertex-specific approach with the one based on graph indices, we grouped the descriptors employed in previous studies of metabolic networks to form a feature vector and analysed its clustering and discriminatory ability. The feature vector comprises of average shortest path length, maximum path length, average clustering coefficient, number of vertices, average vertex degree and average vertex betweenness centrality, each normalised to zero mean and unit variance. Next, we have conducted principal component analysis (PCA). Contrary to results of our vertex-specific descriptors in Fig. 1 the results depicted in the left panel of Fig. 2 do not show meaningful clusters. 

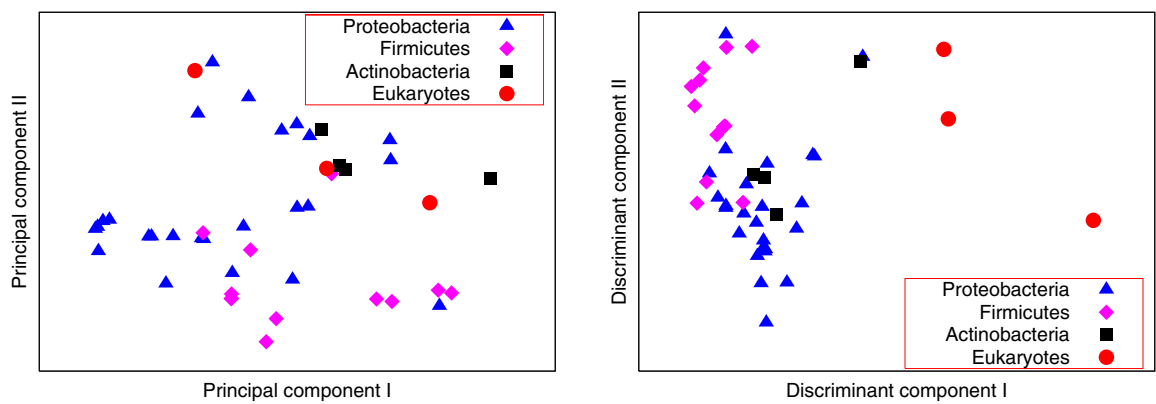

Fig. 2. Principal component space (left) and optimal discriminant space obtained with LDA (right) for a feature set composed of global descriptors and averages of local descriptors of metabolic networks. See text for details.

Lack of clusters, corresponding to groups of organisms, in the principal component space indicates that the main variance captured by graph indices is not associated with inter-group diversity. It does not mean that differences between groups are not captured at all. We evaluated the extent to which analysed graph indices contain information allowing to discriminate between groups of organisms. To this end, we employed a supervised projection method, the Fisher's Linear Discriminant Analysis (LDA) 16, to find an optimal discriminant space. LDA finds a projection optimal in terms of separation between groups, knowing on input the assignment of organisms to groups. The result, depicted in the right panel of Fig. 2, indicates that some discriminatory information is present in the descriptors, at least allowing to separate eukaryotes from bacteria. Unlike for the vertex-specific descriptors, as comparison with PCA results show, this information does not dominate the variance but is overwhelmed by variance within organism groups. It has to be extracted with supervised learning methods that know group assignment from the start, such as LDA. However, such supervised methods are unsuitable for phylogenetic analysis, which is inherently an unsupervised task. Similarly unsatisfactory clustering was obtained when graph indices were computed using only the 100 vertices common to all giant connected components (data not shown), which rules out the possibility that the advantage of our method stems from using only a specific subset of vertices.

For comparison, if the same supervised LDA method is applied to our vertexspecific descriptors based on vertex degrees, as shown in the left panel of Fig. 3 discrimination between groups of organisms is much more evident than for graph indices in Fig. 2. Moreover, clustering in PCA space is not much different from supervised results in LDA space, which further supports the claim that for vertexspecific descriptors, the main source of differences between organisms is related to their evolutionary closeness. As shown in the right panel of Fig. 3, the effectiveness of the vertex-specific approach is not confined to vertex degree only, vertex-specific descriptors based on betweenness centralities of the nodes can also be used. 


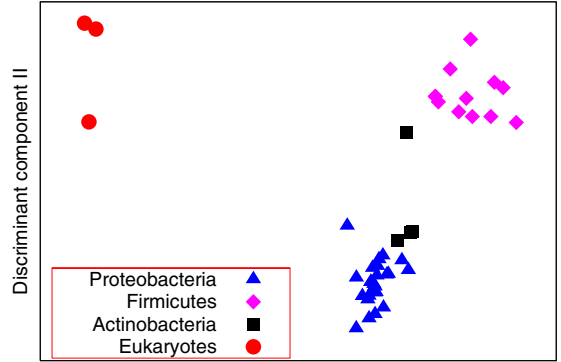

Discriminant component I

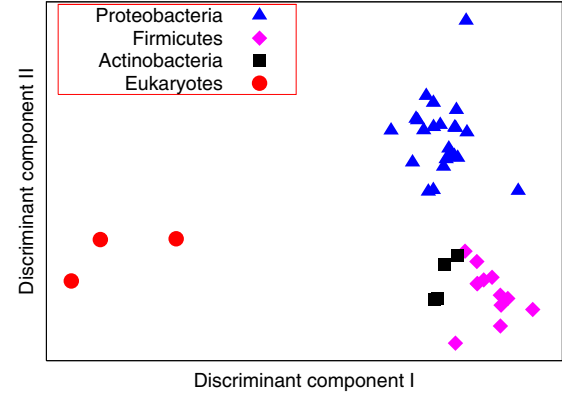

Discriminant component I

Fig. 3. Optimal discriminant space obtained with LDA for the vertex-specific descriptors of metabolic networks, based on vertex degree (left) or vertex centrality (right)

\subsection{Phylogenetic Tree from Vertex-Specific Descriptors of Metabolic Networks}

We have used feature vectors defined by vertex-specific descriptors based on vertex degrees to construct the phylogenetic tree of the 44 analysed organisms.

To this end, we calculated Euclidean distances between normalised feature vectors representing the metabolic networks of the organisms. The distances were then supplied to the NJ phylogenetic tree construction algorithm [17] implemented in the PHYLIP tool. The resulting tree is depicted in Fig. 4.

In the tree, bacteria form a separate subtree from eukaryotes. Within bacteria, a certain amount of taxonomic order is preserved, with the most notable exception being the placement of $N$. meningitidis, a $\beta$-proteobacteria, along with M. leprae, a member of Actinobacteria, and C. crescentus, an $\alpha$-proteobacteria, in a separate subtree. One reason for this may be that $C$. crescentus is a species of Caulobacterales, while all the other $\alpha$-proteobacteria, separated from it in the tree, are Rhizobiales. Furthermore, a recent study using a genome-wide phylogeny indicated that $N$. meningitidis is quite distinct [18. Depending on the tree-construction method, it was situated near $\alpha$ - or $\gamma$-proteobacteria, not along the other $\beta$-proteobacteria with sequenced genome, $R$. solanacearum. In whole-genome phylogeny, $R$. solanacearum aligned itself consistently with a $\gamma$ proteobacteria $P$. aeruginosa, a finding that is also supported by results of our study.

The rest of the organisation of the bacteria tree follows taxonomy, in some groups down to below the level of species class. A subtree of $\alpha$-proteobacteria forms a separate group within Proteobacteria, as do $\gamma$-proteobacteria. Within a common tree of the class of $\gamma$-proteobacteria, a branch corresponding to the order Enterobacteriales is present. Actinobacteria, a phylum of high-GC Grampositive bacteria, and Firmicutes, low-GC Gram-positive bacteria, form a tree isolated from Gram-negative Proteobacteria, with further grouping of Firmicutes into a single branch separated from Actinobacteria. 


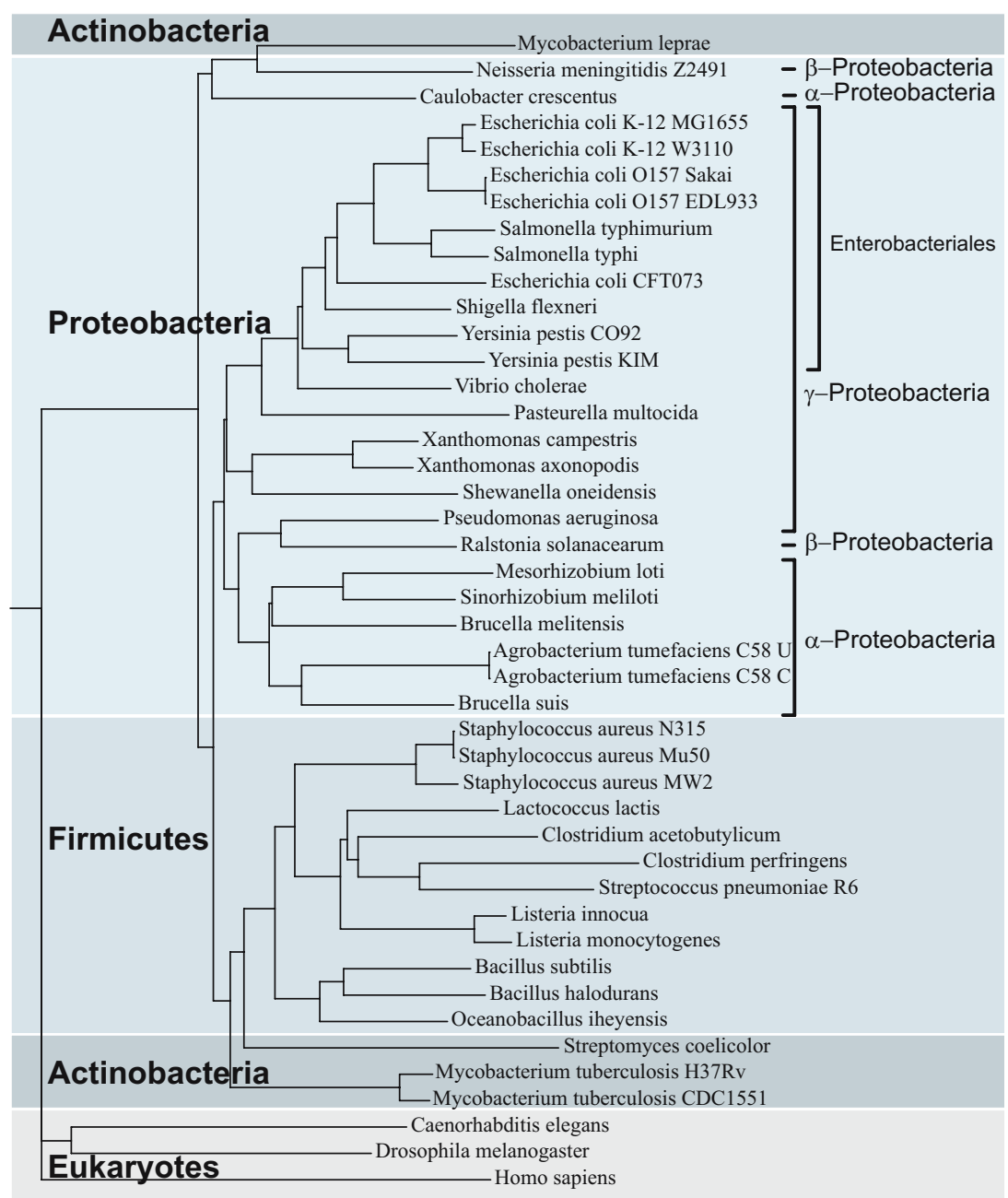

Fig. 4. Phylogenetic tree obtained with NJ tree construction method [17] using the vertex-specific descriptors of metabolic networks based on vertex degrees

\section{Conclusion}

We have shown that the evolutionary distances between organisms can be successfully inferred from simple properties of their metabolic networks. We used the fact that correspondence between vertices in metabolic networks is readily available, as they represent well-defined chemical entities, to construct vertexspecific topological graph descriptors. This network-centred approach allows for capturing evolutionary closeness of organisms at the functional, system level. The resulting clustering of organisms is in most aspects consistent with the well established view that relies on genome content. 
Acknowledgements. The author acknowledges Polish Ministry of Science and Higher Education research projects 3T11F01030 and N519315535 and KI AGH internal grant 11.11.120.777. Graph properties were calculated with Matlab BGL library. The author thanks Prof. Dr. Witold Dzwinel for mentoring, and Mr. Marcin Kurdziel and Mr. Wojciech Czech for discussions.

\section{References}

1. Wolf, Y., Rogozin, I., Grishin, N., Koonin, E.: Genome trees and the tree of life. Trends in Genetics 18, 472-479 (2002)

2. Barabasi, A., Oltvai, Z.: Network biology: understanding the cell's functional organization. Nature Reviews Genetics 5, 101-113 (2004)

3. Jeong, H., Tombor, B., Albert, R., Oltvai, Z., Barabási, A.: The large-scale organization of metabolic networks. Nature 407, 651-654 (2000)

4. Podani, J., Oltvai, Z., Jeong, H., Tombor, B., Barabási, A., Szathmáry, E.: Comparable system-level organization of Archaea and Eukaryotes. Nature Genetics 29, 54-56 (2001)

5. Forst, C., Flamm, C., Hofacker, I., Stadler, P.: Algebraic comparison of metabolic networks, phylogenetic inference, and metabolic innovation. BMC Bioinformatics 7, 67 (2006)

6. Ma, H., Zeng, A.: Phylogenetic comparison of metabolic capacities of organisms at genome level. Molecular Phylogenetics and Evolution 31, 204-213 (2004)

7. Heymans, M., Singh, A.: Deriving phylogenetic trees from the similarity analysis of metabolic pathways. Bioinformatics 19, i138-i146 (2003)

8. Forst, C., Schulten, K.: Phylogenetic analysis of metabolic pathways. Journal of Molecular Evolution 52, 471-489 (2001)

9. Ma, H., Zeng, A.: Reconstruction of metabolic networks from genome data and analysis of their global structure for various organisms. Bioinformatics 19, 270-277 (2003)

10. Zhu, D., Qin, Z.: Structural comparison of metabolic networks in selected single cell organisms. BMC Bioinformatics 6, 8 (2005)

11. Oh, S., Joung, J., Chang, J., Zhang, B.: Construction of phylogenetic trees by kernel-based comparative analysis of metabolic networks. BMC Bioinformatics 7, 284 (2006)

12. Luo, B., Hancock, E.: Structural graph matching using the EM algorithm and singular value decomposition. IEEE Transactions on Pattern Analysis and Machine Intelligence 23, 1120-1136 (2001)

13. Costa, L., Rodrigues, F., Travieso, G., Boas, P.: Characterization of complex networks: A survey of measurements. Advances in Physics 56, 167-242 (2007)

14. Wilson, R., Luo, E.: Pattern vectors from algebraic graph theory. IEEE Transactions on Pattern Analysis and Machine Intelligence 27, 1112-1124 (2005)

15. Ma, H., Zeng, A.: The connectivity structure, giant strong component and centrality of metabolic networks. Bioinformatics 19, 1423-1430 (2003)

16. Bishop, C.M.: Pattern Recognition and Machine Learning. Springer, Heidelberg (2006)

17. Saitou, N., Nei, M.: The neighbor-joining method: a new method for reconstructing phylogenetic trees. Molecular Biology and Evolution 4, 406-425 (1987)

18. Henz, S., Huson, D., Auch, A., Nieselt-Struwe, K., Schuster, S.: Whole-genome prokaryotic phylogeny. Bioinformatics 21, 2329-2335 (2005) 